\title{
THE BONN 408 MHz SURVEY
}

\author{
C. G. T. HASLA M, W. E. WILSON, D. A. GRAHAM, and G. C. HUNT \\ Max-Planck-Institut für Radioastronomie, Bonn, F.R.G.
}

\begin{abstract}
The $100 \mathrm{~m}$ telescope was used to complete the northern sky survey started at Jodrell Bank. The survey aimed at reaching the confusion limit of the telescope. Zero levels are consistent within $\pm 3 \mathrm{~K}$, and scale errors are less than $10 \%$. The survey will be published in the form of maps; a machine readable version is also available.
\end{abstract}

The Bonn $408 \mathrm{MHz}$ survey was designed to fulfil two goals. The first was to provide a simple first experiment for the new $100 \mathrm{~m}$ telescope at Efflesberg. The second purpose was to complete the northern sky survey that was started in 1966 at Jodrell Bank (Haslam et al., 1970) and which was designed to provide data for the study of large scale galactic features such as the loops and subsequently to be used for studies of background spectral distribution.

The Bonn experiment was begun in March 1971 with the construction of a correlation receiver which was specifically designed to have a wide dynamical linear response $(>40 \mathrm{~dB})$ and to have continuous gain measurement. This allowed the survey to be made at high speed without having to reduce the sensitivity as the beam crossed the galactic plane and without having to stop to calibrate the gain. The antenna was designed to accept circular polarization to minimize the errors introduced by the ionosphere in the measurements of the background polarization. The experiment aimed to record a survey down to the confusion limit of the $100 \mathrm{~m}$ telescope. It is essential for survey work that the telescope beam have a low sidelobe level. In this experiment the beam was $37^{\prime}$ with first side lobe $\approx 25 \mathrm{~dB}$ down on the forward response.

As in the Jodrell Bank survey the maps were scanned by nodding scans along the meridian at a speed of $4 \mathrm{deg} \mathrm{min}^{-1}$, the operational upper speed limit of the telescope at the time of the survey. This method has many advantages. The scans intersect many times under repeatable hour angle conditions. It is possible to analyse this data later to work out the receiver baselevel drifts by an iterative process. This method has been used successfully, each hour of right ascention having $\sim 2500$ intersections and requiring about $10 \mathrm{~min}$ of CDC 3300 time to converge to the survey noise level.

Two maps for each region were made using up and down scans separately. These were compared and found to agree to $1.7 \mathrm{~K}$ in the colder regions. The final maps were made by combining these two surveys. The overall temperature calibration was made by convolving the survey down to the resolution of the $404 \mathrm{MHz}$ survey of Pauliny-Toth and Shakeshaft (1962). The comparison showed the zero levels to be consistent to $\pm 3 \mathrm{~K}$ and the scale errors to be less than $10 \%$.

The survey is to be published in Astronomy and Astrophysics Supplement Series in the form of maps. However, we also have the data in machine readable form and have developed a library of computer processing routines which will enable us to 
make maps of selected areas in any coordinate system and beam resolution less than or equal to the original maps. This library will enable us to make contour diagrams as direct overlays for the Palomar Sky Atlas prints. It also allows us to standardize other surveys at other frequencies and resolutions so that direct detailed spectral comparison can be made. We are currently compiling a library of continuum maps for this purpose. An additional use for the survey is to provide maps of the low order terms for high resolution surveys made by the large cross and synthesis telescopes.

At the present time work is in progress on the construction of a new $408 \mathrm{MHz}$ receiver to be used with the Parkes $64 \mathrm{~m}$ telescope to survey the southern sky. The equipment has been extended to allow simultaneous measurements of the total power and linear polarised components of the $408 \mathrm{MHz}$ continuum radiation.

\section{References}

Haslam, C. G. T., Quigley, M. J. S., and Salter, C. J.: 1970, Monthly Notices Roy. Astron. Soc. $147,405$. Pauliny-Toth, I. I. K. and Shakeshaft, J. R.: 1962, Monthly Notices Roy. Astron. Soc. 124, 61.

C. G. T. Haslam

W. E. Wilson

D. A. Graham

G. C. Hunt

Max-Planck-Institut

für Radioastronomie,

Bonn, F.R.G. 